\title{
Parametric Model Of Excavator Working Equipment Based On The Advanced Development Of UG
}

\author{
Minghai Yuan ${ }^{1,2, a}$, Mingxia Kang ${ }^{1,3, b}$, Yue Ding ${ }^{1, c}$ \\ ${ }^{1}$ College of Mechanical and Electrical Engineering, Hohai university, Changzhou,213022,China \\ ${ }^{2}$ Guangxi Key Laboratory for Manufacturing System and Advanced Manufacturing Technology, \\ Nanning, 530004, China. \\ ${ }^{3}$ Huaian Research Institute of Hohai University, Huaian 223001, China \\ aemail: ymhai@126.com, bemail:1005309062@qq.com
} Keywords: The Excavator Working Device; The Advanced Development; Parametric; The 3D
model

\begin{abstract}
The initialized geometric parameters for three main working device components of the hydraulic excavator SY205C - 8s including movable arm, bucket arm and bucket are designed based on relevant design manual. And the geometric models are designed by the application of UG. The design principles followed in the process of initial modeling are after the first, the first time after the main, the surface after the point, the whole after the half. The parametric design platform of excavator working device is established by the secondary development tools of UG/Open API in the secondary development platform of UG.Use the UG/Open Menu Script to design the menu and use the UG/Open UIStyler to design dialog box. In addition, the function of information transfer is realized by the dynamic link library in VC.
\end{abstract}

\section{Introduction}

Hydraulic excavator is a kind of widely used for the construction of the multi-function of construction machinery, as the main models of construction machinery. Because the hydraulic excavator has many varieties, multi-function, high quality and high efficiency etc., thus got the favor of the construction unit, its manufacturing is increasingly booming[1].The working device structure in Different tonnage of excavator are similar, but the size is different. Therefore, in the process of design, we can use the second technology, using the same 3Dmodel, according to the different parameters, to get device modeling done quickly, so as to shorten the design cycle[2].

Parametric design refers to the parts are relatively stereotyped on the shape which use a set of parameters to constraint a set of structure size sequence, through the call and change of geometrical parameters of the models to realize the rapid production and modified of the product model, so as to realize the intelligent product design. Parametric design as the core research contents of CAD technology, has the characteristics of integration, intelligence, is one of the important ways to realize the rapid design of the working equipment of excavator. UG is the most advanced and facing to the manufacturing industry high-end CAD/CAE/CAM software, and it has good openness, using UG/Open secondary development module can develop personalized database applications, can make it more efficient to automatic modeling of standard and special parts, to avoid duplication of effort[3].

\section{The Main Parameters Of Bucket Mechanism Design}

Bucket overall structure is average the longitudinal symmetric structure, can be divided into five parts[4], namely the bucket cavity, bucket lip, bucket teeth, bearing and steel plate parts, welding structure for the overall.Its basic structure as shown in figure 3.1: 
1-side wall 2-side of the blade

3-lateral teeth 4-tooth holder

5-the bucket tooth 6-before the blade

7-the bottom of the bucket

8 - with the bucket rod hinged support

9-the beam 10 - the ear plate

11 - with the connecting rod hinged support

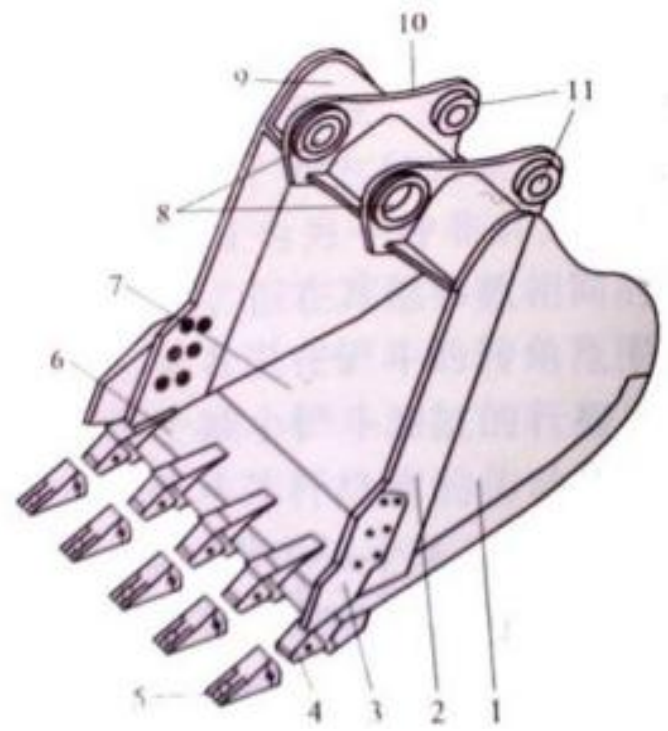

Figure 1 bucket structure

Among them (see figure 2), the main structure design parameters are a bucket capacity of bucket $\mathrm{q}$, turning radius of mining $\mathrm{l} 3$, the average width $\mathrm{b}$, they have the mathematical relationship with the Angle of turning mining as the following:

$$
q=0.5 l_{3}^{2} b\left(2 \varphi_{\max }-\sin 2 \varphi_{\max }\right) K_{s}
$$

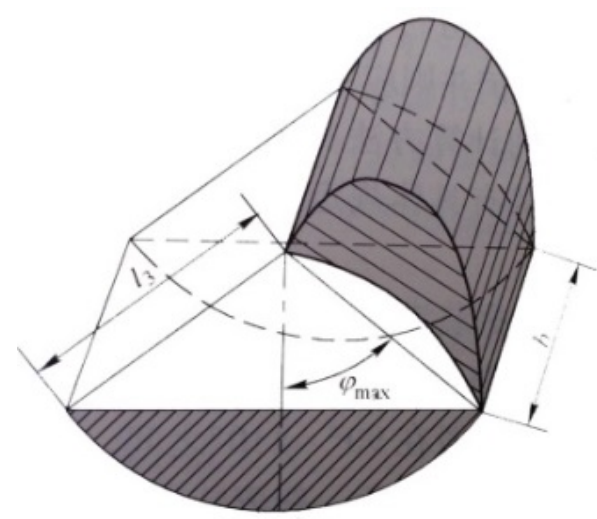

Figure 2 Bucket main parameters

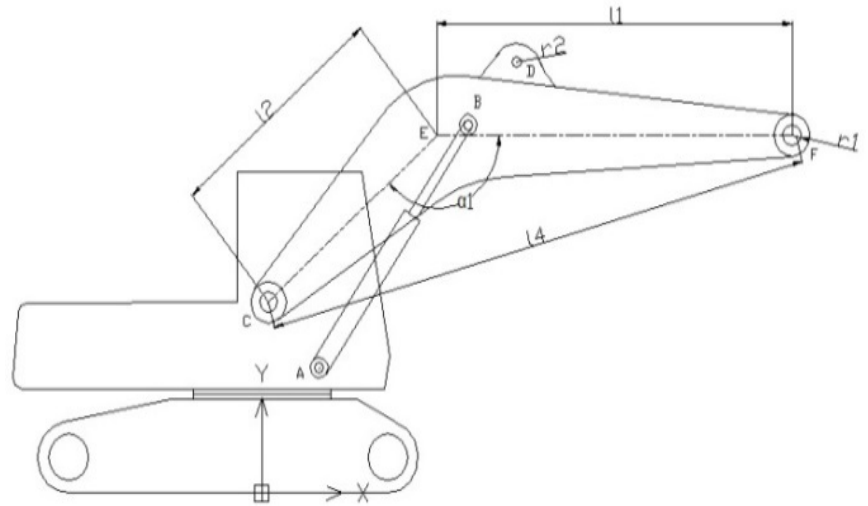

Figure3 Derrick structure parameters design

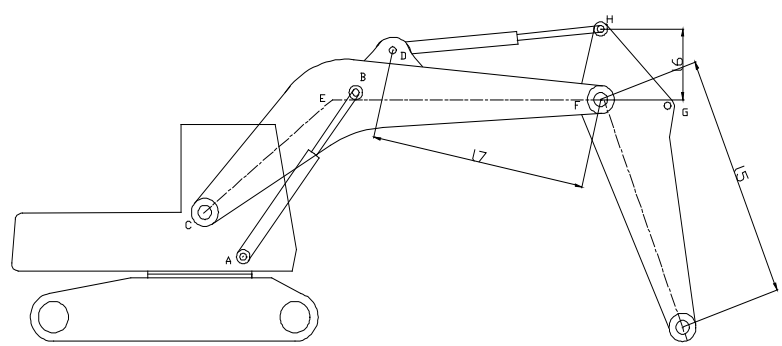

Figure 4 Bucket rod structure parameter design

According to the design requirements, the initial $\mathrm{q}=0.83 \mathrm{~m} 3, \mathrm{l}=1.25 \mathrm{~m}, \mathrm{~b}=1.12$ $\mathrm{m}, 2 \varphi_{\max }=90 \sim 110^{\circ}$, take $2 \varphi_{\max }=100^{\circ}$,Ks as the loose coefficient, associated with the category and their status of soil, primary is 1.25 . Verifying the rationality of selecting the main structural parameters of bucket can be judged according to the turning mining digging capacity, its expression is: 
$E=\frac{K}{b}\left(2 K_{2} \frac{\sin \varphi_{\max }-\varphi_{\max } \cos \varphi_{\max }}{2 \varphi_{\max }-\sin 2 \varphi_{\max }}+100 R K_{3} \frac{\varphi_{\max }-1.5 \sin 2 \varphi_{\max }+2 \varphi_{\max } \cos ^{2} \varphi_{\max }}{2 \varphi_{\max }-\sin 2 \varphi_{\max }}\right)$

Type of $\mathrm{K}$ - thinking other factors influence coefficient in the mining process;

K2-function, when q=0.15-1m3,K2 is1.5;

K3- function, when $\mathrm{q}=0.15-1 \mathrm{~m} 3, \mathrm{~K} 3$ is 0.07 ;

\section{Design The Main Parameters Of Derrick Mechanism}

For backhoe hydraulic excavator, in order to satisfy the maximum digging depth, will usually make movable arm bend shape. Movable arm is consist of the upper and lower two straight and middle curved three segments, as shown in figure 3.

(1)Primary the number of movable arm oil cylinder $\mathrm{n} 1=2$, cylinder $\mathrm{D} 1=150 \mathrm{~mm}$, the piston rod diameter $\mathrm{d} 1=90 \mathrm{~mm}$, the longest length of movable arm cylinder ABmax $=1600 \mathrm{~mm}$, shortest $\mathrm{ABmin}=1000 \mathrm{~mm}$ (scaling $1.6 \leq \lambda_{1}=\frac{A B_{\max }}{A B_{\min }} \leq 1.7$ ), position size $\mathrm{AC}=(0.5-0.6) \mathrm{ABmin}=500 \mathrm{~mm}$;

(2)According to the relevant design standards, the scope of movable arm angle $\alpha_{1}$ is 110 。 140。 for commonly, little movable arm is 150 。 -170 。, the smaller the $\alpha_{1}$, the more severe stress concentration, and increases a lateral force to arise the additional torque, in this paper, taking $\alpha_{1}=140^{\circ}$;

(3)Turn movable arm in bending E divided into two parts. The length ratio of the upper and lower movable arm $k_{2}=\frac{E F}{E C}$ associated with hinged point location B of movable arm cylinder. Primary K2=1.1-1.3, so can primary $11=1750 \mathrm{~mm}, 12=1458 \mathrm{~mm}$. 14 can be obtained from the Pythagorean theorem as shown in type (3) :

$l_{4}=\sqrt{l_{1}^{2}+l_{2}^{2}-2 l_{1} l_{2} \cos \alpha_{1}}=3016 \mathrm{~mm}$

(4)Reference the length ratio of the similar movable arm and the bucket rod, the scope of $k_{1}=\frac{l_{4}}{l_{5}}$ is 1.1-1.3, the value of 15 is $2153 \mathrm{~mm}$, then the biggest radius of excavator working device is designed $r_{1} \approx l_{3}+l_{4}+l_{5} \approx 6419 \mathrm{~mm}$.

\section{The Main Parameters Of The Bucket Rod Mechanism Design}

As the middle parts of the working device, the bucket rod carrying the effect of transmission torque, transforming mining direction, driving the bucket work[5], its structure as shown in figure 4.

(1)Primary the number of bucket rod oil cylinder $\mathrm{n} 2=1$, cylinder diameter $\mathrm{D} 2=150 \mathrm{~mm}$, the piston rod diameter $\mathrm{d} 2=90 \mathrm{~mm}$, the reference range of bucket rod swing angle is $105^{\circ}-125^{\circ}$;

(2)The biggest lever $l_{6}$, also known as the active arm, geometrical relationship can be defined as the distance between bucket rod oil cylinder and the bucket rod hinged point $\mathrm{H}$ with bucket rod and the movable arm hinged point $\mathrm{F}$, according to the torque balance equation, the calculation formula of $l_{6}$ as shown in (4):

$$
l_{6}=\frac{F_{G \max } r_{G 2}}{F_{2}}
$$

Referring to the technical parameters of similar type, arm oil cylinder maximum desirable digging force $F_{G \text { max }}$ can be $50 \mathrm{KN}$, the bucket rod oil cylinder maximum thrust desirable F2 for 225 $\mathrm{KN}$, corresponding resisting arm $r_{G 2}$ is calculated by the status of the shovel bucket lip and the cutter movement curve tangent :

$$
r_{G 2}=l_{3} \cos \left(\frac{\pi}{2}-\beta\right)+\sqrt{\left(l_{5}^{2}-\left(l_{3} \sin \left(\frac{\pi}{2}-\beta\right)\right)^{2}\right)}=l_{3} \sin \beta+\sqrt{\left(l_{5}^{2}-\left(l_{3} \cos \beta\right)^{2}\right)}
$$


In the type, $\beta$ is generally about $60^{\circ}$.

By type (4) (5) can calculate: $r_{G 2}=3672 \mathrm{~mm}, l_{6}=816 \mathrm{~mm}$;

(3)The telescopic of Bucket rod of oil cylinder is relevant with the scope of swinging angle $\theta_{2 \max }$ and $l_{6}$,

$$
\begin{aligned}
& \Delta D H=D H_{\max }-D H_{\min } \approx\left(\lambda_{2}-1\right) D H_{\min }=2 l_{6} \sin \frac{\theta_{2 \max }}{2} \\
& D H_{\max } \approx \frac{2 \lambda_{2} l_{6} \sin \frac{\theta_{2 \max }}{2}}{\lambda_{2}-1} D H_{\min } \approx \frac{2 l_{6} \sin \frac{\theta_{2 \max }}{2}}{\lambda_{2}-1} \\
& \text { By type } \quad(6) \quad(7) \quad \text { can } \quad \text { calculate: DHmax } \quad=1900 \mathrm{~mm}, \quad \text { DHmin } \\
& =1145 \mathrm{~mm} \text {,scaling } 1.6 \leq \lambda_{2}=\frac{D H_{\max }}{D H_{\min }} \leq 1.7 .
\end{aligned}
$$

(4)When the bucket rod oil cylinder to the longest, according to the geometrical relationship to determine the position of the articulated point $\mathrm{D}$ of the bucket rod oil cylinder $\mathrm{t}$ on the moving arm, the expression as shown in (8):

$$
l_{7} \approx \sqrt{l_{6}^{2}+D H_{\max }^{2}-2 l_{6} D H_{\max } \sin \frac{\theta_{2 \max }}{2}} \approx 1235 \mathrm{~mm}
$$

When the bucket rod oil cylinder to the shortest, the formula (9) can be used to check the rationality of $l_{7}$ :

$$
D H_{\min }+l_{6}>l_{7}
$$

\section{The Parametric Design Of Working Device}

In this paper, the software platform of UG and VC + + is used to realize the parametric design of excavator working device, the design process mainly include the work device of the UG secondary development and the design of $\mathrm{VC}++$ work device instance library two aspects. In the process of secondary development, use UG to establish $3 \mathrm{~d}$ model of each part of the working equipment[6], in the process of modeling, add the corresponding geometric constraints and characteristics constraint to the initialize model, to realize the control for the model structure shape. And then through the extract of the main parameters of the related modules of the design models or directly call parameters from the database, achieve rapid modeling of product. The extraction of the related parameter of parts module is the key to the parametric design of the work device, the realization of the process not only need to design a series of interrelated expressions in the process of establishing $3 \mathrm{~d}$ model initialization, still need through the UG/API to design related parameter extraction control program and the ODBC data interface program to realize the parameters passing and calls. At the same time using database technology to redact the geometry parameter database of work device parts models, through the edit of the geometrical parameters of the model and the calls of the data in the database, in order to realize the automatic invocation and update of each product parts information. The concrete design scheme is shown in figure 5.

\section{UG Secondary Development Process}

Based on the UG NX 6.0 as the platform of the parameterized modeling of excavator working device, the key technologies of realizing the parametric design is reasonably build the parametric model and the parametric driving mechanism of the design objects. In UG parameterized modeling can be realized by two methods:

One is using the secondary development tools brought by UG itself namely the UG/Open Grip module, to design the Grip language program, the user needed to directly driver program to generate models automatically generated according to the demand, called a fully programmed 
parametric modeling.But if change the model design data need to start from changing the process, extremely inconvenient.

Second, using UG/Open API module to development and design, developers by the program of UG/Open API, and through the $\mathrm{C}$ or $\mathrm{C}++$ programming to call these functions, establish the MFC dialog, the designer can through input parameters in the dialog box to obtain the demand models directly, this method is called parameters of the modified method of modeling, but must have a support of product instance library by using the method. This article using is the parameters of the modified method of modeling, the method not only simplifies the process of $3 \mathrm{D}$ modeling of the excavator working equipment, and embodies the advantages of the modular technology well. Its development process is shown in figure 6.

First create a folder Startup and folder Application under the main folder, and then use the MenuScript to design the main menu for the system application program, and generate the file*.menu, the menu file saved to the folder Startup, at the same time apply UIStyler to edit dialog, and save the generated files *.DLG, ${ }^{*} \mathrm{~h},{ }^{*} \mathrm{c}$ to the folder Application. Again into the VC compiler environment create a Win32 Dynamic Link Library project to realize the callback of the function dialog box, in the process of compiling ,first should change file *.c under the folder Application into file *.CPP, then *. H and *. CPP files are copied to the VC project directory at the same time, and then carries on the compilation of the function, finally save the generated Dynamic Link Library files * DLL to the file Startup,then the secondary development of UG/Open API can be completed.

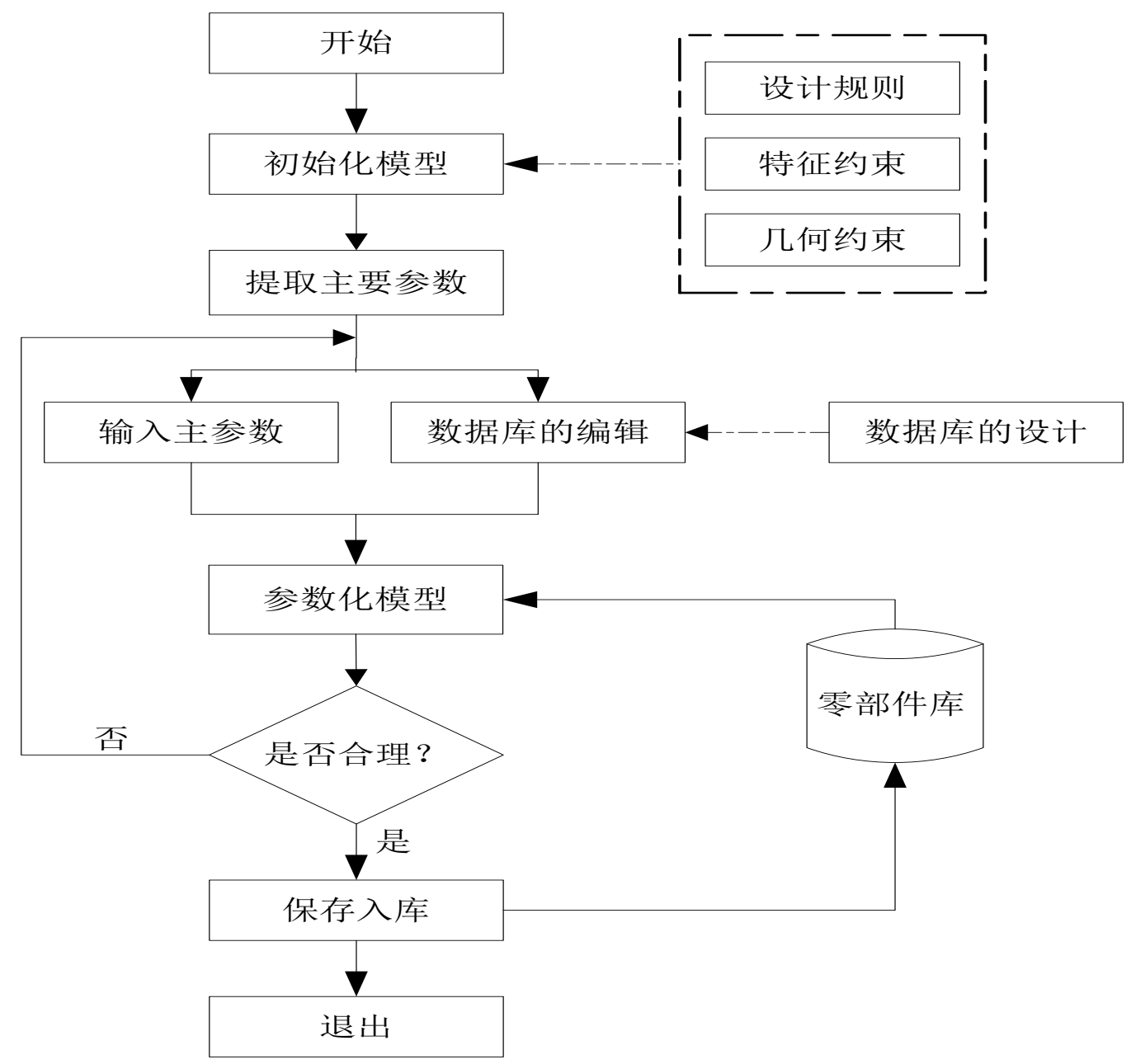

Figure 5 The parametric design of excavator working device 


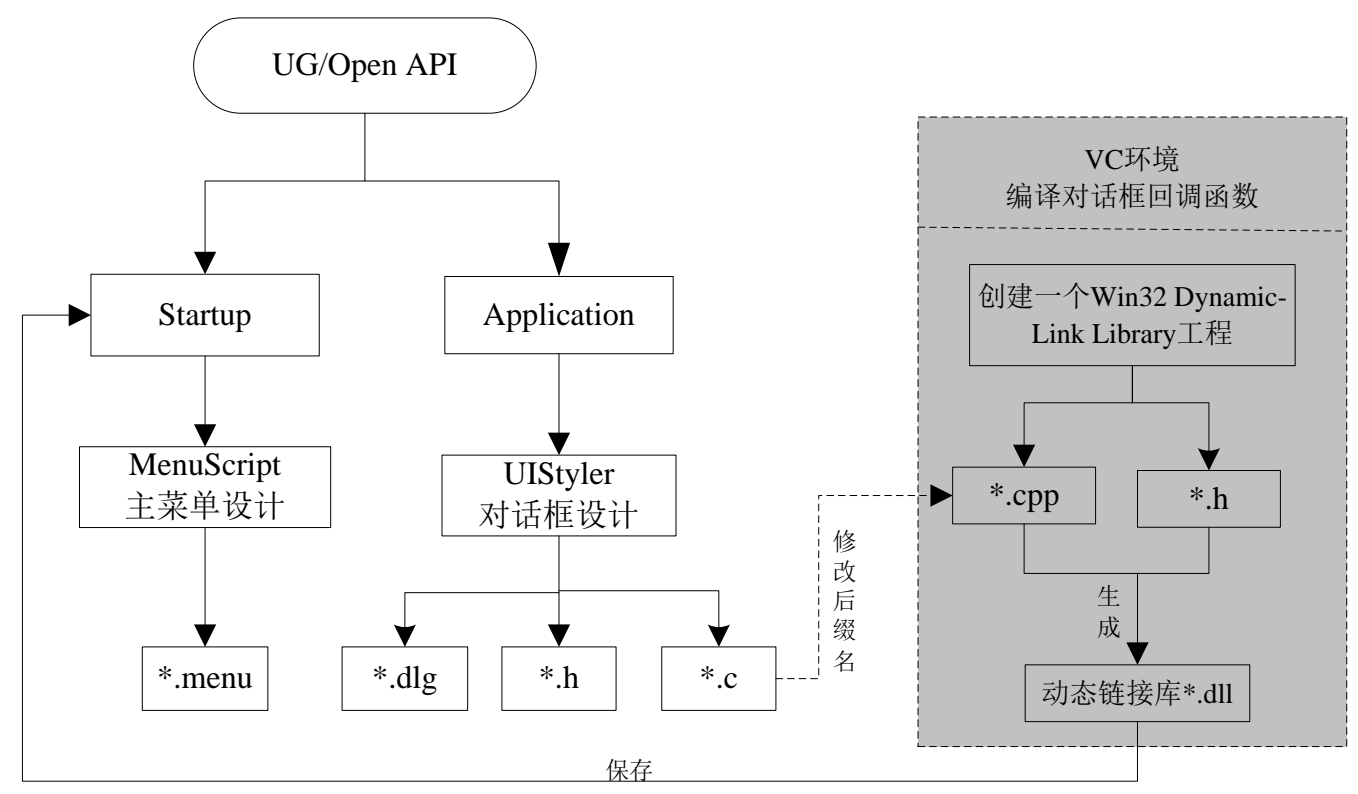

Figure 6 The secondary development process based on UG/Open API

\section{The Realization Of A Parameterized Modeling}

Based on the UG/Open API for parametric design, including the design principles in the initial process of modeling, they are after home on time after the first, first point behind, after the first side blocks. First determine the articulated point position, again through the constraint of point to establish the contour of each plate, tensile the face consisted of lines to obtain plate solid model, through the combination of various kinds of sector entities and detail treatment, model simplification, finally obtain the physical model by using the mirror. Upon completion of the modeling of the initial model, the design of the parametric dialog box and the preparation of a link program, the most important and the most critical step is must to register environment variables firstly, connecting all the development platforms with UG itself. Through the "my computer"'"properties" edit variables called UGII_USER_DIR, variable values are working directory for the system design.

Through the UG menu to open the initial models need parameterized, through modeling dialog, according to user needs to change the related design parameters can obtain the model of an instance that user need.

\section{Conclusion}

This paper take the hydraulic excavator SY205C - $8 \mathrm{~s}$ as the design model, do a initialized geometric parameters design for the movable arm,bucket arm and bucket the three main components of excavator working device according to relevant design manual,and then apply UG to design geometric models. In the secondary development platform of UG, the parametric design platform of excavator working device is established by the secondary development tools of UG/Open API. In addition, use the dynamic link library in VC to realize information transfer.

\section{Acknowledgements}

This work was supported by the Applied Basic Research Programs of Changzhou under grant number CJ20140027, the open fund of Huaian Research Institute of Hohai University, and theopen fund ofGuangxi Key Laboratory for manufacturing system and advanced manufacturing technology under Grant number 13-051-09S09, andthe graduate student innovation project of Changzhou campus of hohai university. 


\section{Reference}

[1] Yuchen Chen.The parametric design and the simulation analysis of small hydraulic excavator working device[D].Sichuan.Southwest jiaotong university.2010.

[2] Degang Guo,Fusheng Zhang. Parametric Model of Excavator Working Equipment Based on the Advanced Development of Pro/E [J].Journal of taiyuan science and technology.2008.7(2)79-81.

[3] Weixing Wen and soon.The parametric design and research on excavator hydraulic cylinder[J].Journalof mechanical engineering and automation.116(3)71-73.

[4] Jiahong Zhao.The mathematical model of hydraulic excavator working mechanism design [J]. Woodworking machine.2014(1)112-116.

[5] Dragan Ignjatovic, Branko Petrovic,Predrag Jovancic. Impact of the Bucket Wheel Support at Technical Parameters of the Block and Bucket Wheel Excavator Capacity[J]. Proceedings of the 12th International Symposium Continuous Surface Mining - Aachen 2014Lecture Notes in Production Engineering 2015.(4)73-81.

[6] Jun Gu. Improved control of intelligent excavator using proportional-integral-plus gain scheduling [D].Seward in Journal of Central South University.2012. 